Canadian

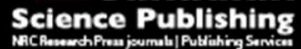

Canadian Journal of Physiology and Pharmacology Revue canadienne de physiologie et pharmacologie

\title{
Thermal and Cytokine Responses to Endotoxin Challenge During Early Life
}

\begin{tabular}{|r|l|}
\hline Journal: & Canadian Journal of Physiology and Pharmacology \\
\hline Manuscript ID & cjpp-2017-0167.R2 \\
\hline Date Submitted by the Author: & Brief Report \\
\hline Complete List of Authors: & $\begin{array}{l}\text { McDonald, Fiona; University of Calgary Cumming School of Medicine } \\
\text { Khawaja, Ahmad; University of Calgary Cumming School of Medicine } \\
\text { Imran, Ahmad; University of Calgary Cumming School of Medicine } \\
\text { Ellis, Margot; University of Calgary Cumming School of Medicine } \\
\text { Chandrasekharan, Kumaran; University of Calgary Cumming School of } \\
\text { Medicine } \\
\text { Hasan, Shabih; University of Calgary }\end{array}$ \\
\hline $\begin{array}{r}\text { Is the invited manuscript for } \\
\text { consideration in a Special } \\
\text { Issue?: }\end{array}$ & N/A \\
\hline Keyword: & $\begin{array}{l}\text { Inflammation, Fever, Cytokines, Neonatal, Sudden Infant Death Syndrome } \\
\text { (SIDS) }\end{array}$ \\
\hline \multicolumn{2}{|c}{} \\
\hline
\end{tabular}




\section{During Early Life}

4 Fiona B. McDonald ${ }^{1}$, Ahmad M. Khawaja ${ }^{2}$, Ahmad A. Imran ${ }^{2}$, Margot E. Ellis ${ }^{2}$, Kumaran Chandrasekharan ${ }^{2}$, Shabih U. Hasan ${ }^{2 *}$ Medicine, University of Calgary, Alberta, Canada.

$8 \quad{ }^{2}$ Department of Pediatrics, Alberta Children's Hospital Research Institute, Faculty of Medicine, University of Calgary, Alberta, Canada.

*Corresponding Author:

University of Calgary

123330 Hospital Drive N.W.

13 Calgary, Alberta,

14 Canada. T2N 4N1

Tel: 403-220-3741

16 Email: hasans@ucalgary.ca

Running Head: Lipopolysaccharide and Neonatal Cytokine Response 
ABSTRACT:

21 Sudden infant death syndrome (SIDS) remains the leading cause of infant mortality beyond the

22 neonatal period. An increase in body temperature as a result of high environmental temperature,

23 over-wrapping of infants and/or infection are associated with SIDS. Endotoxins such as

24 lipopolysaccharide (LPS) and heat stress may perturb cardiorespiratory function and

25 thermoregulation. Although LPS-mediated body temperature and cytokine responses are well-

26 documented in older animals, the capacity of LPS to induce fever and cytokine response in

27 young rats remains unclear. Therefore, we sought to investigate the acute effects of LPS on body

28 temperature and cytokine concentrations in rat pups. Postnatal day 7 rat pups were divided into

29 three groups. Group 1 was administered LPS intraperitoneally $(200 \mu \mathrm{g} / \mathrm{kg})$. Group 2 received

30 saline at volume equal to LPS group. Group 3 received no treatment. Pups were placed in

31 custom-made chambers maintained at ambient temperature of $33^{\circ} \mathrm{C}$. Body surface temperature

32 was continuously monitored for four hours. Thereafter, the rats were euthanized and serum

33 collected for cytokine analysis. We demonstrate that LPS treatment increased MIP-1 $\alpha$, IL-10,

34 MCP-1, IP-10, fractalkine and TNF- $\alpha$ with no concurrent rise in body surface temperature.

35 Although neonatal rats produced an array of cytokines in response to LPS, there was no evidence 36 of fever.

37 Key Words: Inflammation, Fever, Sudden Infant Death Syndrome (SIDS), Neonatal, Cytokines 


\section{INTRODUCTION}

Sudden infant death syndrome (SIDS), defined as the sudden death of an infant that remains unexplained after a thorough case investigation, including performance of a complete autopsy, examination of the death scene and review of the clinical history (Moon et al. 2007). An increase in body temperature as a result of high environmental temperature, over-wrapping of infants and/or infection are associated with SIDS. Similarly, prone sleeping position that may interfere with heat dissipation is the single most important extrinsic risk factor for SIDS. Since the successful advocacy of "back to sleep" position, the rates of SIDS have markedly decreased, yet SIDS remains the leading cause of infant death beyond the neonatal period (Moon et al. 2007).

Antigens such as lipopolysaccharide (LPS), once recognized by antigen presenting cells, stimulate an inflammatory response in the host through the actions of multiple cytokines via prostanoid dependent and independent pathways (Blatteis et al. 2000; Fabricio et al. 2006; Roth and De Souza 2001; Zampronio et al. 2015). Inflammatory responses may cause fever, i.e. an increase in body temperature in response to change in thermoregulatory set point (Zampronio et al. 2015). However, body temperature may also be unnaturally increased by high ambient temperature due to overheating the dwelling and/or overwrapping of infants resulting in hyperthermia. Although a number of well-designed studies have investigated the role and mechanisms of fever and cytokine responses in the context of LPS administration in adult rodents including rats (Koenig et al. 2014; Long et al. 1990; Luheshi and Rothwell 1996; Machado et al. 2007; Roth and De Souza 2001; Rummel et al. 2011a), to our knowledge, limited data are available during early life (Fraifeld and Kaplanski 1998). Thus, it is critical to elucidate if LPS administration leads to increased body temperature and/or increased circulating pro- 
60 inflammatory cytokines in neonatal rats and whether it is possible to delineate the effects of

61 infection per se versus increased body temperature caused by fever. Recent studies from our

62 laboratory have investigated inflammatory responses to LPS administration in young rat pups,

63 however, the animals were also exposed to hypoxia and restraint stress (McDonald et al. 2016a;

64 McDonald et al. 2016b); both factors may impact the inflammatory responses (Doherty and

65 Blatteis 1980; Fournier et al. 2015; Ricciuti and Fewell 1992). Elucidating the effects of LPS on

66 body surface temperature and cytokine responses is the first step in quantifying the interaction of

67 LPS-mediated cytokine and thermal responses. Therefore, the specific aims of this study were: 1)

68 to elucidate if LPS administration affects body temperature over time within a controlled thermal

69 environment and 2) to investigate the LPS-induced cytokine response in one week old rat pups.

70 We hypothesized that LPS administration would result in inflammatory cytokine response with

71 concurrent rise in body temperature over time.

\section{MATERIALS and METHODS}

73 Experiments were performed on 24 Sprague-Dawley rat pups from 8 litters on postnatal day 7.

74 There were three experimental groups; Control, Saline and LPS. Each group comprised of pups

75 from 8 litters. LPS was administered intraperitoneally (IP) at $200 \mu \mathrm{g} / \mathrm{kg}$. Saline was administered

76 also IP at a volume equivalent to LPS injection. Control group received no treatment. No pup

77 was studied on more than one occasion and pups for various experimental groups were randomly

78 selected from the entire cohort of pregnant rats. A thermocouple was affixed to a cohesive

79 bandage (Co-Flex, Andover, MA) as described previously (McDonald et al. 2016a; McDonald et

80 al. 2016b). The pup was then placed in a custom-made double-walled glass chamber through

81 which water circulated from a water bath, to maintain an ambient temperature of $33^{\circ} \mathrm{C}$, 
82 considered within thermoneutral range for a rat pup (Mortola 2001). The heated water circulated

83 through the double-wall lumen without being in contact with the animals. The chambers were open-ended but covered loosely with parafilm punched with multiple holes to allow rat pups to

85 breathe room air. Three experiments were performed in parallel with three chambers connected in series. Temperature in each chamber was monitored using a thermocouple. Ambient chamber

87 temperature $\left(T_{c}\right)$ and body surface temperature $\left(T_{B S}\right)$ were recorded continuously and noted every 15 min for four hours. The duration of the study was chosen as previous studies have demonstrated an increase in body temperature during this period (Luheshi 1998; Machado et al. 2007; Roth and De Souza 2001). At the end of the experiment, rats were euthanized and blood samples were obtained, spun down and serum stored at $-80^{\circ} \mathrm{C}$ for cytokines analysis. The timeinterval between placement of rat pups in thermal chambers and/or saline/LPS injection, and blood sampling for cytokines analysis was four hours.

Cytokine analysis was performed by Eve Technologies, located at the University of Calgary, 95 using the Rat Cytokine Array/Chemokine Array 27-Plex. The multiplex assay was performed using the Bio-Plex ${ }^{\mathrm{TM}} 200$ system (Bio-Rad Laboratories), and a Milliplex rat cytokine kit

97 (Millipore) according to their protocol. The 27-plex consisted of EGF, Eotaxin, Fractalkine, GCSF, GM-CSF, GRO/KC/CINC-1, IFN $\gamma$, IL-1 $\alpha$, IL-1 $\beta$, IL-2, IL-4, IL-5, IL-6, IL-10, IL-12(p70), IL-13, IL-17A, IL-18, IP-10, Leptin, LIX, MCP-1, MIP-1 $\alpha$, MIP-2, RANTES, TNF $\alpha$ and VEGF.

Temperature measurements and cytokine data are presented as mean $\pm \mathrm{SD}$. Tc and $\mathrm{T}_{\mathrm{BS}}$ data were analyzed using two- way ANOVA (LPS treatment $\mathrm{x}$ time). Cytokine data were tested for outliers using Grubbs test, maximum of one outlier was removed per group $\mathrm{P}<0.05$. Cytokine data were 
103 then analyzed using one-way ANOVA (LPS treatment). Tukey's post-hoc test was performed if

104 ANOVA reached significance $(\mathrm{P}<0.05)$.

105 RESULTS

106 Animals

107 Body Mass

108 The body mass of the rat pups was not different between the three groups before the experiment;

109 (Control 16.2 \pm 5.3 ; Saline injection 16.61 \pm 2.3 and LPS injection $16.9 \pm 2.5 \mathrm{~g} ; p=0.84$ ).

110 Thermal chamber $\left(T_{C}\right)$ and body surface temperature $\left(T_{B S}\right)$

111 The $\mathrm{T}_{\mathrm{C}}$ temperature remained constant throughout the experiment and each chamber was within

$1120.2{ }^{\circ} \mathrm{C}$ (Figure 1$) . \mathrm{T}_{\mathrm{BS}}$ was plotted against time and while the temperature increased slightly over 113 four hours in all groups (control, saline or LPS; $1{ }^{\circ} \mathrm{C}, 1.13{ }^{\circ} \mathrm{C}$ and $1.5{ }^{\circ} \mathrm{C}$ respectively). The 114 treatment was not a significant factor (treatment $p=0.7$, time $\mathrm{p}<0.0001$ and interaction $p=0.9$, 115 Figure 1).

\section{Cytokines}

117 The serum concentrations of 27 cytokines/chemokines $(\mathrm{pg} / \mathrm{ml})$ and statistical analysis are 118 presented in Table 1 and Figure 2. Fractalkine $(p<0.0001)$, IL-10 $(p=0.003)$, IP-10 $(p=0.002)$, 119 MCP-1 $(p=0.01), \operatorname{MIP}-1 \alpha(p=0.01)$ and TNF $\alpha(p=0.0003)$ concentrations were significantly 120 higher in the LPS treatment group compared with those observed in both saline and control 121 groups (Figure 2). IL-17A ( $p=0.006)$ had higher concentrations in both saline and LPS groups 122 compared with the control group values, however, these values were similar between saline- and 123 LPS-treated groups (Table 1). Overall, IL-6 and MIP-2 serum concentrations were higher in the 
124 LPS-treated group but did not reach statistical significance $(p>0.05$; Table 1$)$. Non-significant 125 increases were observed in $\mathrm{EGF}$ and $\mathrm{GRO} / \mathrm{KC}$ concentrations (Table 1). The remainder of the 126 cytokine concentrations were equivalent between the groups.

\section{DISCUSSION}

128 We demonstrate that body temperature remains stable over time in a thermoneutral environment 129 and while LPS increased serum concentrations of cytokines, it did not significantly increase body 130 temperature. To our knowledge, this is the first study providing a large profile of cytokine 131 concentrations in both naïve and LPS-treated rat pups. The data demonstrate that an LPS-induced 132 change in cytokine concentrations can be independent of an increase in body surface temperature 133 in one week old rat pups. We provide the much needed baseline data on thermal and cytokine 134 responses to LPS that might form the basis of mechanistic studies on inflammation and 135 temperature regulation during early life.

136 Evidence suggests that febrile response including the biphasic pattern is highly variable and 137 dependent on species, sex, age, homeothermia, ambient temperature, dose of endotoxin and its 138 route of administration, and prior antigenic exposure among others (Boissé et al. 2004; Fraifeld 139 and Kaplanski 1998; Kluger et al. 1998; Rudaya et al. 2005; Zhao et al. 2008). Although fever is 140 a hallmark of infection in adults, there are mixed reports on whether neonatal and some adult 141 mammals develop fever in response to infection and whether newborns are capable of mounting 142 a sufficient immune response (Gervassi and Horton 2014; Zhao et al. 2008). Fraifeld and 143 Kaplanski (1998) summarized the pyrogenic responses at various postnatal ages and reported 144 that 3 to 14 day old rats did not develop fever and the newborns in contrast to the weaning rats 145 had prolonged hypothermic response. However, the studies were performed at $22^{\circ} \mathrm{C}$ ambient 
146 temperature which is well below $\left(>10^{\circ} \mathrm{C}\right)$ the thermoneutral range. Given that the newborns of 147 smaller species are unable to maintain their body temperature, the prolonged hypothermia likely 148 reflected the ambient temperature rather than the effects of LPS treatment (Fraifeld and 149 Kaplanski 1998).

Although the mechanisms of absence of LPS-associated fever in young animals remain unknown, several possibilities exist. Since cytokines interactions can be both synergistic and inhibitory, very high TNF- $\alpha$ concentrations observed in our study might have prevented the febrile response (Long et al. 1990). Furthermore, a dissociation between the rise of cytokines and initial phase of fever has been attributed to pyrogenic signals. It is, thus, possible that our naïve young animals lacked pre-formed pyrogenic factor and complement fragments (Roth and De Souza, 2001). Also, we did not observe an increase in leptin concentrations which has been postulated as one of the pyrogenic cytokines (Koenig et al. 2014). Though we have consistently observed increased levels of TNF- $\alpha$, simultaneous IL-10 concentrations also increased following LPS challenge in our current and other studies (McDonald et al. 2016a; McDonald et al. 2016b). Endotoxin-induced fever in adult rats is associated with a significant rise of IL-10 (Tavares et al. 2005). IL-10 consistently increased in response to LPS in adult mice (Erickson and Banks 2011). In fact, IL-10 deficient mice exhibit exacerbated and prolonged fever in response to LPS (Kluger et al. 1998). Similar to findings in neonatal infants, serum cytokine samples show little TLR4mediated IL-12p70 production IL-12(p70) but a high IL-10 response and therefore not strongly promoting a pro-inflammatory effect. It is likely, given the presence of cytokines in circulation in our study that neonatal animals can detect antigens/pyrogens, however there may be a selflimiting process to fever generation through anti-pyretic modulation or perhaps a blunted response at the level of the anterior hypothalamus. The most recent understanding of the innate 
169 immune system is that it is preferentially polarized to an anti-inflammatory state, which in theory

170 may leave young mammals susceptible to infection, as the body does not mount a sufficient

171 inflammatory response to overcome the infection.

172 In adults, Toll-like receptor (TLR) activation initiates the process of cytokine production that

173 triggers an innate immune response that predominately comprises of macrophages, monocytes

174 and neutrophils. The acute phase response is the period in which cytokines, neutrophils and

175 hormones are released into the plasma and lead to centrally coordinated sickness behavior in

176 adults. Fever occurs alongside sickness behavior in adults and is a robust indicator of infection

177 (Blatteis et al. 2000). Fever in adult rats occurs ultimately as the result of an upregulation of 178 prostaglandin E (PGE) at the level of the ventromedial preoptic-anterior hypothalamus, however 179 the mechanism that leads to this remains under debate and more than one route and mediator are 180 possible. The puzzling fact about fever is that the rapid onset is unlikely to be mediated either by 181 cytokines which need to be synthesized de novo or other humoral factors interfacing at the blood 182 brain barrier which also need de novo (cyclooxygenase 2) COX2 and PGE signaling (Blatteis et 183 al. 2000). Thus, it is likely that a neural mechanism leads to fever mediated via vagal afferents 184 from the liver (complement, C5a) activation of Kupffer cells to release PGE2 causing 185 norepinephrine release that activates constitutive COX2 and therefore rapid release of PGE2 as 186 evidenced by studies on adult guinea-pig and rats but this too remains under debate (Fabricio et 187 al. 2006; Li et al. 2006). Both pre- and full-term infants can increase their body temperature in 188 response to PGE1 administration thus indicating that infants are responsive to PGs and absence 189 of fever cannot be attributed to decreased sensitivity or lack of pyrogenic effects of PGs during 190 early neonatal period (Heymann and Clyman 1982). 
191 Although neural and humoral factors mediate the rapid onset of fever, there is a strong role for 192 cytokine mediators in fever such as IL-6, TNF- $\alpha$, IL-1 $\beta$, MIP $1 \alpha$ and MIP-1 in adult rats 193 (Fabricio et al. 2006; Zampronio et al. 2015). In adult rats, IL-6 was found to mediate fever 194 response through mPEGS-1 in the brain and IL-6 deficient adult mice are resistant to fever and 195 do not demonstrate an increase in $\mathrm{PGE}_{2}$. TNFa is produced primarily by mast cells and functions 196 to recruit monocytes, dendritic cells and polymorphonuclear cells. TNF- $\alpha$ is produced in 197 response to endotoxin (Karck et al. 1988) and induces fever in adult rats (Stefferl et al. 1996). 198 Our study shows an absence of fever to a moderate dose of LPS but a clear activation of 199 pyrogenic cytokine production (eg.IL-6 and TNF- $\alpha$ ). Nonetheless, IL-6 is known to have diverse 200 pyrogenic capacities dependent on the concentration and species (Rummel et al. 2011b). In 201 young rats, IL-6 played a strong role in fever genesis while TNF $\alpha$ modulated the initial 202 pyrogenic phase but was not necessary for the later phase of LPS-mediated rise in body 203 temperature (Harden et al. 2006). Thus, fever response may be dependent on expression of other 204 immune mediators. Similar to the current study, IP-10, MIP-1 $\alpha$, MCP-1 also increased in adult 205 mice in response to LPS (Erickson and Banks 2011; Kopydlowski et al. 1999). Furthermore 206 MCP-1 is also upregulated in adult rats following LPS administration (Uchiumi et al. 2004), 207 though may not be directly pyrogenic (Zampronio et al. 2015). There was no observed LPS208 induced increase in GRO (CXCL1) in our study, which is a mediator of prostaglandin dependent 209 fever in adult rats (Soares et al. 2008). Moreover, IL-1 $\beta$ was also not increased following LPS 210 treatment, though in adult rats IL-1 $\beta$ upregulation is small and more short-lived compared to 211 other cytokines (Kakizaki et al. 1999).

212 In our study, a small but significant increase in IL-17A concentrations was observed in both 213 LPS- and saline-treated animals. These changes might reflect the injection-related stress response 
214 as mild thermal stress response has previously been reported (Rummel et al. 2011a). These 215 changes also highlight the significant impact of intraperitoneal injection on cytokine responses 216 regardless of the injected agent (vehicle and LPS in this case). It also brings up the point that in 217 studies where no naïve (control) group is included, the impact of various 218 manipulations/interventions on at least some of the cytokine responses might be at risk of under219 or over-estimation. An LPS-mediated increase in IL-17A has previously been documented by 220 Sun et al. (2015), however, the administered dose $(500 \mu \mathrm{g} / \mathrm{kg})$ was more than twice what we 221 used in our study.

222 Immunity comes at a high metabolic cost and may be an evolutionary adaptation to minimize 223 immune response over initial few days of growth and development. Fever is part of an acute, 224 centrally co-ordinated response which mediates a change in the thermoregulatory set point which 225 must be achieved by minimizing heat loss (vasoconstriction) or by heat generation that can be 226 accomplished by an increase in metabolic heat production (shivering or non-shivering 227 thermogenesis and decrease in heat loss) (Zampronio et al. 2015). Fraifeld and Kaplanski (1998) 228 suggest actual body temperature may not always reflect the functional state of the central 229 thermostat and increased $\mathrm{PGE}_{2}$ production in hypothalamus would not directly lead to body 230 temperature elevation in rodents.

231 Our study has some limitations. Although we provide foundational data on a large number of 232 cytokines and demonstrate dissociation between fever and increased pro-inflammatory cytokines, 233 our study does not provide mechanisms underlying such observations. Furthermore, inclusion of 234 adult animals might have provided interesting comparative physiological data. Measurement of 235 rectal or intraabdominal temperature is a gold standard. In our study, we measured body surface 
236 temperature as surrogate for rectal temperature. These measurements will not reflect body

237 temperature in older animals, however may be considered valid in very young rat pups as body

238 temperature at this age closely follows the ambient temperature which was kept within

239 thermoneutral range (Mortola 2001). 
241 In summary, we provide new observations on serum cytokine concentrations in naïve animals

242 and those exposed to a moderate LPS dose. Furthermore, we demonstrate that despite immune

243 activation by LPS, there was no corresponding rise in body temperature of one week old rat

244 pups. Thus, it is possible to delineate the effects of mild infection per se versus increased body

245 temperature caused by fever. 
246 Ethics: The University of Calgary Animal Care Committee approved the study protocols and all 247 experimental procedures followed the national guidelines set by the Canadian Council on Animal 248 Care.

249 Authors' Contribution: F.B.M, A.K, K.C and S.U.H designed the study. Experiments were 250 performed by A.K and K.C. F.B.M. A.K, A.I, M.E, S.U.H reviewed the data and performed 251 analysis. All authors participated in the manuscript preparation and final approval.

252 Competing Interests: None.

253 Funding: SIDS Calgary Society.

254 Acknowledgments: We thank Professor Quentin Pittman for his technical advice during the 255 studies and Ms. Patricia Hyatt and Ms. Shylin Miljan for administrative assistance. 


\section{REFERENCES}

Blatteis, C.M., Sehic, E., and Li, S. 2000. Pyrogen sensing and signaling: old views and new concepts. Clinical infectious diseases: an official publication of the Infectious Diseases Society of America. 31 Suppl 5: S168-177. doi: 10.1086/317522.

Boissé, L., Mouihate, A., Ellis, S., and Pittman, Q.J. 2004. Long-Term Alterations in Neuroimmune Responses after Neonatal Exposure to Lipopolysaccharide. The Journal of Neuroscience. 24(21): 4928.

Doherty, D.J., and Blatteis, C. 1980. Hypoxic reduction of endotoxic fever in guinea pigs. J Appl Physiol Respir Environ Exerc Physiol. 49(2): 294-299.

Erickson, M.A., and Banks, W.A. 2011. Cytokine and Chemokine Responses in Serum and Brain After Single and Repeated Injections of Lipopolysaccharide: Multiplex Quantification with Path Analysis. Brain, Behavior, and Immunity. 25(8): 1637-1648. doi: 10.1016/j.bbi.2011.06.006.

Fabricio, A.S.C., Rae, G.A., Zampronio, A.R., D'Orléans-Juste, P., and Souza, G.E.P. 2006. Central endothelin $\mathrm{ET}_{\mathrm{B}}$ receptors mediate IL-1-dependent fever induced by preformed pyrogenic factor and corticotropin-releasing factor in the rat. American Journal of Physiology - Regulatory, Integrative and Comparative Physiology. 290(1): R164-R171. doi: 10.1152/ajpregu.00337.2005.

Fournier, S., Gulemetova, R., Baldy, C., Joseph, V., and Kinkead, R. 2015. Neonatal stress affects the aging trajectory of female rats on the endocrine, temperature, and ventilatory responses to hypoxia. American Journal of Physiology - Regulatory, Integrative and Comparative Physiology. 308(7): R659-R667.

Fraifeld, V., and Kaplanski, J. 1998. Brain eicosanoids and LPS fever: species and age differences. Progress in brain research. 115: 141-157.

Gervassi, A.L., and Horton, H. 2014. Is Infant Immunity Actively Suppressed or Immature? Virology : Research and Treatment. 5: 1-9. doi: 10.4137/VRT.S12248.

Harden, L.M., du Plessis, I., Poole, S., and Laburn, H.P. 2006. Interleukin-6 and leptin mediate lipopolysaccharide-induced fever and sickness behavior. Physiology \& Behavior. 89(2): 146-155. doi: http://dx.doi.org/10.1016/j.physbeh.2006.05.016.

Heymann, M.A., and Clyman, R.I. 1982. Evaluation of Alprostadil (Prostaglandin E1) in the Management of Congenital Heart Disease in Infancy. Pharmacotherapy: The Journal of Human Pharmacology and Drug Therapy. 2(3): 148-154. doi: 10.1002/j.1875-9114.1982.tb04522.x.

Kakizaki, Y., Watanobe, H., Kohsaka, A., and Suda, T. 1999. Temporal Profiles of Interleukin-1.BETA., Interleukin-6, and Tumor Necrosis Factor- $\alpha$. in the Plasma and Hypothalamic Paraventricular Nucleus after Intravenous or Intraperitoneal Administration of Lipopolysaccharide in the Rat. Estimation by Push-Pull Perfusion.:Estimation by Push-Pull perfusion. Endocrinologia Japonica. 46(4): 487-496. doi: 10.1507/endocrj.46.487.

Karck, U., Peters, T., and Decker, K. 1988. The release of tumor necrosis factor from endotoxinstimulated rat Kupffer cells is regulated by prostaglandin $\mathrm{E}_{2}$ and dexamethasone. Journal of Hepatology. 7(3): 352-361. doi: 10.1016/S0168-8278(88)80008-4. 
Kluger, M.J., Kozak, W., Conn, C.A., Leon, L.R., and Soszynski, D. 1998. Role of Fever in Disease. Annals of the New York Academy of Sciences. 856(1): 224-233. doi: 10.1111/j.17496632.1998.tb08329.x.

Koenig, S., Luheshi, G.N., Wenz, T., Gerstberger, R., Roth, J., and Rummel, C. 2014. Leptin is involved in age-dependent changes in response to systemic inflammation in the rat. Brain Behav Immun. 36: 128-138. doi: 10.1016/j.bbi.2013.10.019.

Kopydlowski, K.M., Salkowski, C.A., Cody, M.J., van Rooijen, N., Major, J., Hamilton, T.A., and Vogel, S.N. 1999. Regulation of Macrophage Chemokine Expression by Lipopolysaccharide In Vitro and In Vivo. The Journal of Immunology. 163(3): 1537.

Li, Z., Perlik, V., Feleder, C., Tang, Y., and Blatteis, C.M. 2006. Kupffer cell-generated $\mathrm{PGE}_{2}$ triggers the febrile response of guinea pigs to intravenously injected LPS. American Journal of Physiology Regulatory, Integrative and Comparative Physiology. 290(5): R1262-R1270. doi: 10.1152/ajpregu.00724.2005.

Long, N.C., Otterness, I., Kunkel, S.L., Vander, A.J., and Kluger, M.J. 1990. Roles of interleukin 1 beta and tumor necrosis factor in lipopolysaccharide fever in rats. American Journal of Physiology Regulatory, Integrative and Comparative Physiology. 259(4): R724.

Luheshi, G., and Rothwell, N. 1996. Cytokines and fever. International archives of allergy and immunology. 109(4): 301-307.

Luheshi, G.N. 1998. Cytokines and Fever: Mechanisms and Sites of Action. Annals of the New York Academy of Sciences. 856(1): 83-89. doi: 10.1111/j.1749-6632.1998.tb08316.x.

Machado, R.R., Soares, D.M., Proudfoot, A.E., and Souza, G.E. 2007. CCR1 and CCR5 chemokine receptors are involved in fever induced by LPS (E. coli) and RANTES in rats. Brain Res. 1161: 21-31. doi: 10.1016/j.brainres.2007.05.054.

McDonald, F.B., Chandrasekharan, K., Wilson, R.J.A., and Hasan, S.U. 2016a. Cardiorespiratory control and cytokine profile in response to heat stress, hypoxia, and lipopolysaccharide (LPS) exposure during early neonatal period. Physiological Reports. 4(2): e12688. doi: 10.14814/phy2.12688.

McDonald, F.B., Chandrasekharan, K., Wilson, R.J.A., and Hasan, S.U. 2016b. Interactive effects of maternal cigarette smoke, heat stress, hypoxia, and lipopolysaccharide on neonatal cardiorespiratory and cytokine responses. American Journal of Physiology Regulatory, Integrative and Comparative Physiology. 311(6): R1113-R1124. doi: 10.1152/ajpregu.00062.2016.

Moon, R.Y., Horne, R.S.C., and Hauck, F.R. 2007. Sudden infant death syndrome. The Lancet. 370(9598): 1578-1587. doi: http://dx.doi.org/10.1016/S0140-6736(07)61662-6.

Mortola, J.P. 2001. Respiratory Physiology of Newborn Mammals; A Comparative Perspective. John Hopkins University Press, Baltimore.

Ricciuti, F., and Fewell, J.E., 1992. Fever in young lambs: hypoxemia alters the febrile response to a small dose of bacterial pyrogen. J Dev Physiol. 17(1): 29-38. PMID: 1645013 
Roth, J., and De Souza, G.E. 2001. Fever induction pathways: evidence from responses to systemic or local cytokine formation. Brazilian journal of medical and biological research $=$ Revista brasileira de pesquisas medicas e biologicas. 34(3): 301-314.

Rudaya, A.Y., Steiner, A.A., Robbins, J.R., Dragic, A.S., and Romanovsky, A.A. 2005. Thermoregulatory responses to lipopolysaccharide in the mouse: dependence on the dose and ambient temperature. American Journal of Physiology - Regulatory, Integrative and Comparative Physiology. 289(5): R1244-R1252. doi: 10.1152/ajpregu.00370.2005.

Rummel, C., Gerstberger, R., Roth, J., and Hubschle, T. 2011a. Parthenolide attenuates LPS-induced fever, circulating cytokines and markers of brain inflammation in rats. Cytokine. 56(3): 739-748. doi: 10.1016/j.cyto.2011.09.022.

Rummel, C., Matsumura, K., and Luheshi, G.N. 2011b. Circulating IL-6 contributes to peripheral LPS-induced mPGES-1 expression in the rat brain. Brain research bulletin. 86(5-6): 319-325. doi: 10.1016/j.brainresbull.2011.09.006.

Soares, D.M., Machado, R.R., Yamashiro, L.H., Melo, M.C.C., and Souza, G.E.P. 2008. Cytokine-induced neutrophil chemoattractant (CINC)-1 induces fever by a prostaglandin-dependent mechanism in rats. Brain Research. 1233: 79-88. doi:https://doi.org/10.1016/j.brainres.2008.07.069.

Stefferl, A., Hopkins, S.J., Rothwell, N.J., and Luheshi, G.N. 1996. The role of TNF- $\alpha$ in fever: opposing actions of human and murine TNF- $\alpha$ and interactions with IL- $\beta$ in the rat. British Journal of Pharmacology. 118(8): 1919-1924. doi: 10.1111/j.1476-5381.1996.tb15625.x.

Sun J., Zhang S., Zhang X., Zhang X., Dong, H., and Qian, Y. 2015. IL-17A is implicated in lipopolysaccharide-induced neuroinflammation and cognitive impairment in aged rats via microglial activation. Journal of Neuroinflammation. 12:165. doi:10.1186/s12974-015-0394-5.

Tavares, E., Maldonado, R., Ojeda, M.L., and Miñano, F.J. 2005. Circulating Inflammatory Mediators during Start of Fever in Differential Diagnosis of Gram-Negative and Gram-Positive Infections in Leukopenic Rats. Clinical and Diagnostic Laboratory Immunology. 12(9): 1085-1093. doi: 10.1128/CDLI.12.9.1085-1093.2005.

Uchiumi, D., Kobayashi, M., Tachikawa, T., and Hasegawa, K. 2004. Subcutaneous and continuous administration of lipopolysaccharide increases serum levels of triglyceride and monocyte chemoattractant protein-1 in rats. Journal of Periodontal Research. 39(2): 120-128. doi: $10.1111 / \mathrm{j} .1600-0765.2004 .00716 . x$.

Zampronio, A.R., Soares, D.M., and Souza, G.E.P. 2015. Central mediators involved in the febrile response: effects of antipyretic drugs. Temperature. 2(4): 506-521. doi: $10.1080 / 23328940.2015 .1102802$.

Zhao, J., Kim, K.D., Yang, X., Auh, S., Fu, Y.-X., and Tang, H. 2008. Hyper innate responses in neonates lead to increased morbidity and mortality after infection. Proceedings of the National Academy of Sciences. 105(21): 7528-7533. 


\section{$371 \quad$ Legends}

$372 \quad$ Figure 1

373 Body surface (circles) and ambient chamber temperature (squares) of control (open), saline

374 (grey) and LPS (solid) groups over the duration of the experiment.

375 Figure 2

376 Group data (mean \pm SD) illustrating serum cytokine concentrations ( $\mathrm{pg} / \mathrm{ml}$ ) of P7 neonatal rat

377 pups in control, saline and LPS groups. ${ }^{*} P<0.05$ LPS vs. both control and saline group.

378 
379 Table 1. Serum cytokine concentrations ( $\mathrm{pg} / \mathrm{ml})$.

\begin{tabular}{|c|c|c|c|c|}
\hline n & $\begin{array}{c}\text { Control } \\
8\end{array}$ & $\begin{array}{l}\text { Saline } \\
\qquad 8\end{array}$ & $\begin{array}{c}\text { LPS } \\
8\end{array}$ & $\begin{array}{c}\text { One-way ANOVA } \\
\text { P }\end{array}$ \\
\hline IL-1 $\alpha$ & $83 \pm 81$ & $67 \pm 65$ & $157 \pm 149$ & 0.2 \\
\hline GCSF & $32 \pm 35$ & $39 \pm 43$ & $32 \pm 35$ & 0.9 \\
\hline GMCSF & $78 \pm 12$ & $85 \pm 14$ & $83 \pm 16$ & 0.59 \\
\hline Leptin & $14763 \pm 10585$ & $10385 \pm 2032$ & $9000 \pm 7408$ & 0.32 \\
\hline IL-4 & $9 \pm 5$ & $8 \pm 4$ & $8 \pm 3$ & 0.76 \\
\hline IL-1及 & $217 \pm 186$ & $509 \pm 712$ & $240 \pm 231$ & 0.38 \\
\hline IL-2 & $33 \pm 24$ & $19 \pm 13$ & $25 \pm 18$ & 0.41 \\
\hline IL-6 & $87 \pm 89$ & $92 \pm 82$ & $3286 \pm 4709$ & 0.04 \\
\hline EGF & $0.014 \pm 0.013$ & $0.016 \pm 0.014$ & $7.1 \pm 13$ & 0.14 \\
\hline IL-13 & $0.2 \pm 0.22$ & $0.11 \pm 0.1$ & $0.10 \pm 0.2$ & 0.49 \\
\hline IL-12 (p70) & $7.3 \pm 2.7$ & $9.4 \pm 6.3$ & $9.7 \pm 6.4$ & 0.67 \\
\hline IFNy & $72 \pm 72$ & $74 \pm 72$ & $185 \pm 253$ & 0.28 \\
\hline IL-5 & $23.5 \pm 13.3$ & $29.8 \pm 14.0$ & $31.8 \pm 20.0$ & 0.57 \\
\hline IL-17A & $7.4 \pm 5.8$ & $17.2 \pm 5.9 *$ & $15.4 \pm 5.4 *$ & 0.006 \\
\hline IL-18 & $11750 \pm 3608$ & $12687 \pm 6929$ & $9101 \pm 4031$ & 0.37 \\
\hline GRO/KC & $225 \pm 227$ & $224 \pm 241$ & $18281 \pm 33288$ & 0.14 \\
\hline VEGF & $221 \pm 20$ & $224 \pm 32$ & $223 \pm 66$ & 0.99 \\
\hline LIX & $4072 \pm 849$ & $3927 \pm 698$ & $4212 \pm 1118$ & 0.82 \\
\hline MIP2 & $124 \pm 103$ & $122 \pm 103$ & $1238 \pm 1655$ & 0.04 \\
\hline Rantes & $7493 \pm 1994$ & $8376 \pm 2720$ & $7110 \pm 2659$ & 0.59 \\
\hline Eotaxin & $10 \pm 10$ & $11 \pm 10$ & $10 \pm 7$ & 0.91 \\
\hline
\end{tabular}

Mean $\pm S D .{ }^{*} P<0.05$ LPS and Saline groups vs. control group. 


\section{Temperature over time}

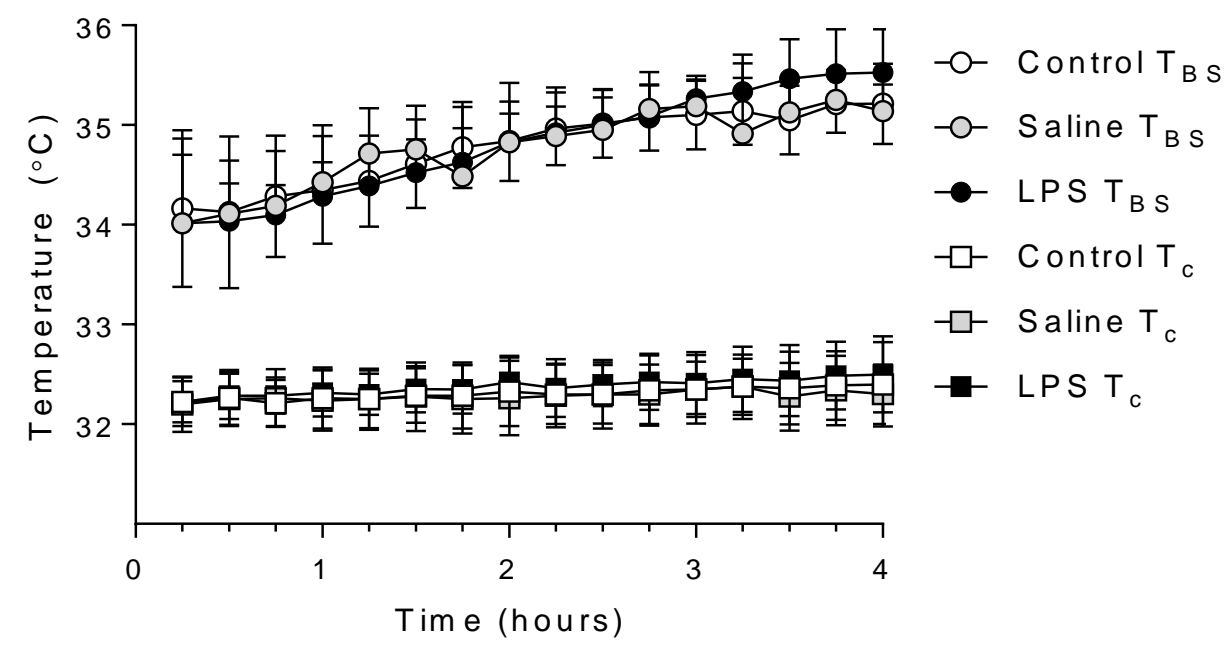

Figure 1 


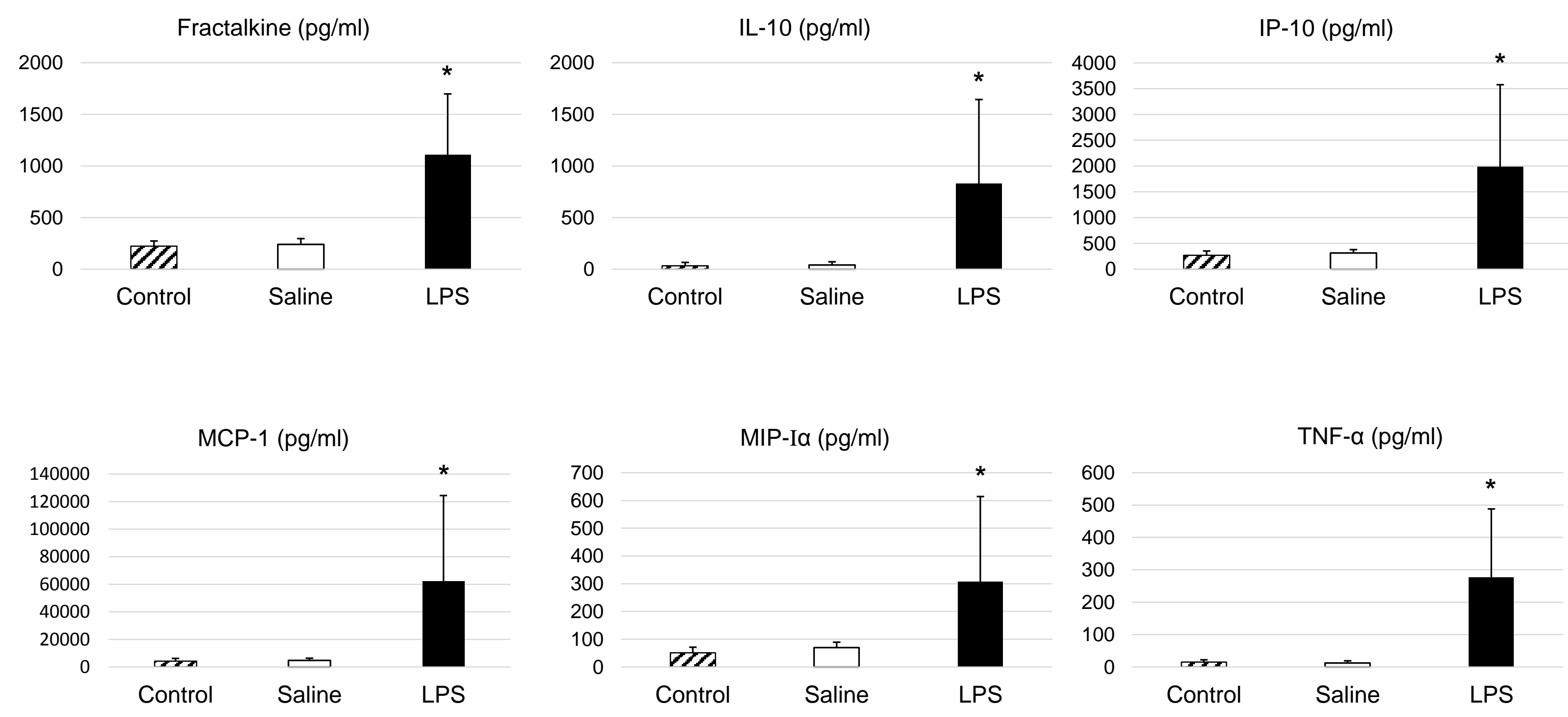

